\title{
EVALUATION OF TOPOLOGY OPTIMIZATION AND GENERATIVE DESIGN TOOLS AS SUPPORT FOR CONCEPTUAL DESIGN
}

\author{
D. Vlah ${ }^{凶}$, R. Žavbi and N. Vukašinović \\ University of Ljubljana, Slovenia \\ $\bigotimes$ daria.vlah@fs.uni-lj.si
}

\section{Abstract}

Nowadays, a large number of different tools that support early phases of design are available to engineers. In the past decade a specialized set of CAD-based tools were developed, that support the ideation process by generating different design alternatives according to the criteria given by the designer. Two types of tools are discussed in this paper: topology optimization and generative design tools. To investigate to what extent these tools are suitable for use in early design phases and what are the main differences between them, a study was conducted on an industrial case.

Keywords: design tools, design optimisation, conceptual design, generative design, topology optimization

\section{Introduction}

Conceptual design is a fuzzy front-end phase of the product development process in which engineers and designers are generating and evaluating multiple design alternatives in order to find the most optimal solutions. Decisions made in this stage will have a strong impact on all the following product development phases which involve different areas such as manufacturing, production, testing, finance and other (Wang et al., 2002). Anderson (2014) argues that up to $80 \%$ of the product costs can be determined by the end of the design phase. Therefore, in the design phase multiple requirements have to be considered to prevent accumulation of additional costs in later development phases.

In order to provide support to creativity, to enable faster generation and testing of the concepts and to speed up the process, engineers have developed various design tools. Recent advancements in technology, especially in the field of computing power, machine learning and related algorithms have set grounds to the development of intelligent design automation tools. High-performance computing power available via cloud now enables complex calculations, optimisations and iterations, that was impossible to perform before. Engineers can now run the advanced simulations to test various product configurations in different conditions in a short time, which gives them information of a great significance for decision making. On the other side, rapid development of the additive manufacturing technologies is leading to significant changes in manufacturing and design of components. They enable production of complex geometries that better fit the design requirements, that were not possible to be produced with traditional manufacturing methods (Bici et al., 2017). Additionally, new materials are being engineered, which have better properties and are compatible with the new manufacturing methods. We are experiencing a gradual shift of design support systems to conceptual design (Horváth, 2004), especially in the way of how CAD tools are applied in the design process. Until the recent emergence 
of tools that can generate designs in CAD based on the algorithms, engineers have been applying a traditional design workflow where CAD tools have been used to only implement design ideas, but not to develop them. The aforementioned technological advancements have led to the increased interest in digital design generation and optimization tools, whose results were usually lightweight structures constructed as complex organic shapes. In the early 2000s engineers have started developing CADbased topology optimization tools that were capable of generating optimal designs of the given structures. Such tools are able to modify existing designs, incorporate explicit features into a design, and generate completely new designs; however, this has mostly been appreciated only by structural designers and engineers, and not by the wider field of product design (Querin, 2017). This concern has recently been addressed by the introduction of generative design tools in CAD software. Main aim of these tools is to support designers' creativity by generating multiple design alternatives. This was achieved by adding the possibility of multiple material and manufacturing selection in a single design study and a different design approach.

Aim of this work is to explore the capabilities of topology optimization and generative design tools and to define the advantages, drawbacks and differences between them in order to conclude which tools would be more suitable for use in the conceptual design phase. To test the capabilities of the tools, a design study was conducted on a race car suspension component (a rocker).

Based on their capability to generate multiple optimal design alternatives by simultaneously considering different material and manufacturing options, we argue that generative design tools might provide a better support in early design phases.

\section{Topology optimization}

Topology optimization is a computational design method which aim is to optimize the distribution of material in a given design space with respect to the loads and constraints, while maximizing the design's performance (Bendsøe, 1989; Bendsøe and Kikuchi, 1988; Querin, 2017). It is typically used in the early design phase to explore and evaluate numerous design possibilities according to the set objectives, such as minimizing the weight, maximizing stiffness, reducing stress, reducing displacement etc (Querin, 2017). Topology optimization tools are developed to reduce the efforts of engineers required for performing the iterative processes of designing and analysing multiple design variations, as well as to support creativity be offering solutions that engineers maybe wouldn't have thought of.

Topology optimization is one of the three main categories of structural optimization, along with shape and size optimization. Shape optimization considers specified parameters of contours defined by the positions of nodes in order to satisfy assigned design criteria and objectives (e.g. reduction of stress concentrations or increasing fatigue life). Size optimization varies the values of design parameters related to cross-sectional areas of the elements in order to find optimum solution regarding weight, stress, displacements etc. The latter is often applied to the problems concerning truss structures, support bars and construction frames. Unlike topology optimization, these methods do not allow for addition or removal of new elements or voids in the structure, only a change of values of their parameters (Querin, 2017; Tejani et al., 2018). Moreover, shape and size optimization methods require having an initial parametrized model to perform the optimization, whereas topology optimization only requires a defined starting volume. The focus of our work was on exploration of tools that can provide optimal design solutions in the conceptual phase when dimensions and shape features are still not defined, therefore we have devoted our investigation to topology optimization method.

There are numerous topology optimization methods based on various algorithms, which can be deterministic or stochastic. Stohastic algorithms are more suitable for the search of multiple solutions because of the element of randomness in their process, which makes them a better fit for conceptual phase (Meyer and Vajna, 2018). In algorithms objective functions are usually defined as minimisation or maximisation functions for the given design parameters. Every optimisation algorithm has to include at least one objective to navigate the design process, which is the case of a single-objective optimization (SOA). It often happens that there are multiple objectives that construct a problem definition. Objectives can be conflicting, resulting with no single optimal solution for the problem, but a plethora of solutions, which all satisfy the given criteria differently. A method used to search for solutions when multiple objectives (criteria) have to be met is called multi-objective optimisation 
(MOO). This method is very convenient to be applied for design problems, which often happen to have contrasting requirements. One of the most straightforward examples of conflicting requirements is lowering the cost and increasing the user safety, which is typically expressed through a choice of materials, stiffness and other properties. The aim of MOO is to simultaneously include all the criteria in the search to provide solutions that have the best trade-offs among all the relevant objectives. Naturally, these solutions will bring an improvement in one or a few aspects and will worsen in others, but are still highly ranked in the solution space. A set of points that have equal trade-off optimal solutions, that cannot be improved in any of the objectives without degrading at least one of the others, is called a Pareto-optimal front (Deb, 2011).

The most commonly implemented methods for topology optimization are: the solid isotropic material with penalization (SIMP) (Bendsøe, 1989; Rozvany, 2000) as an element-based approach, discrete approaches such as evolutionary structural optimization method (ESO) (Xie and Steven, 1994), level set method (LSM) (Jia et al., 2011) and combined approaches.

Many of the commercial topology optimization tools use the SIMP method, which is mesh-dependent, meaning it requires a starting geometry for a study that is a closed volume (solid body). Regarding functionality in these softwares, they are mostly capable of single objective optimization or multi objective optimization that is reduced to a single objective by implementation of weights. To explore Pareto optimal front by finding multiple optimized results, one would have to perform a number of iterations of this process by changing the values of study parameters, such as materials or target objectives.

\section{Generative design}

Research on generative design began around 1980s, but at the time it included mostly publications that were purely theoretical, without any notable example of application. With the forthcoming development of computers and technology, researchers have started to search for possible solutions that could utilize these new resources to improve their working processes. The biggest initial interest was shown in the field of architecture (Caldas, 2001; Shea et al., 2005), but soon after, the researchers from other fields have started to investigate possible opportunities and applications which the combination of computing and analogies drawn from the evolution theory could offer.

In the field of engineering design Vajna et al. (2005) have introduced Autogenetic Design Theory where they have investigated the similarities of the design process as a part of the product development process and the natural process of evolution. They state that "Product development process can be described from the evolutionary view as a continuous optimization of a basic solution by observing starting conditions, boundary conditions and constraints". These factors influence the design evolution and put up the design space. They can be expressed as orders, customer requirements, spontaneous ideas, guidelines etc. and are possible to change during the process, which is analogous to the changes in environment found in nature. Up until today, because of its rather large area of applications, there is still no commonly accepted definition for generative design. Shea et al. (2005) described the aim of generative design as following: "generative design systems are aimed at creating new design processes that produce spatially novel yet efficient and buildable designs through exploitation of current computing and manufacturing capabilities. Krish (2011) offered more narrow definition for generative design, focusing on its implementation in CAD: "Generative design is a designer driven, parametrically constrained design exploration process, operating on top of history based parametric CAD systems structured to support design as an emergent process". However, today there are applications in engineering design that are not bound to parametric models and those that surpass the use of standard CAD programs, e.g. (Chen et al., 2018; Kazi et al., 2017).

Generative design can be understood as a method for creating multiple designs, which includes certain amount of automation and autonomy in the process. Nature's evolutionary approach is applied to design process, starting with one or a number of different designs which are spread all over the design space, which evolve with time into more suitable forms to given conditions. Designs which do not fit to the conditions or do not follow the design goals are discarded, and the evolving process continues to navigate in other directions. Engineers and designers are involved in the process primarily to set the constraints and design goals before the generation starts, but could also be included, as well as the 
customers, to interfere throughout the whole generation process. Even though generative design can be done just with a pen and paper using a defined set of rules, the term is typically used for computationally aided design. The generated outputs can take various forms: images, models, sounds, animation etc., hence this method can be applied in different fields like architecture, engineering and design, art, music, fashion and many others.

Generative design is typically related to the use of algorithms as a basis for design creation. Recently generative design tools have been introduced as separate modules in a number of commercial CAD softwares for engineering design. Generative design tools are initially based on algorithms used in topology optimization, namely the level set method (LSM) (Jia et al., 2011). They operate with moving boundaries instead of local density variables, therefore they have the ability to be meshindependent (Tyflopoulos et al., 2018) thus having different requirements for design setup than topology optimization. LSM is characterized by flexibility and capability to handle demanding topological changes. Similarities and differences of topology optimization and generative design tools will be discussed in the following sections.

\section{Case study}

With the aim of exploring capabilities and finding differences among the topology optimization and generative design approaches we have conducted a study on a race car crank bell lever design, which is commonly known as a rocker. Rocker is one of the components that form a race car suspension system, which is in our case directly influenced by a force from a pushrod that is counterbalanced by a spring rod and an anti-roll bar (ARB) drop link force (Figure 1). To simplify the design task by reducing it to two-dimensional load case, a single rocker plate was considered rather than the whole rocker assembly. The initial model which was used as a basis geometry for both topology optimization and generative design was taken from a real industrial race car design (Figure 1).
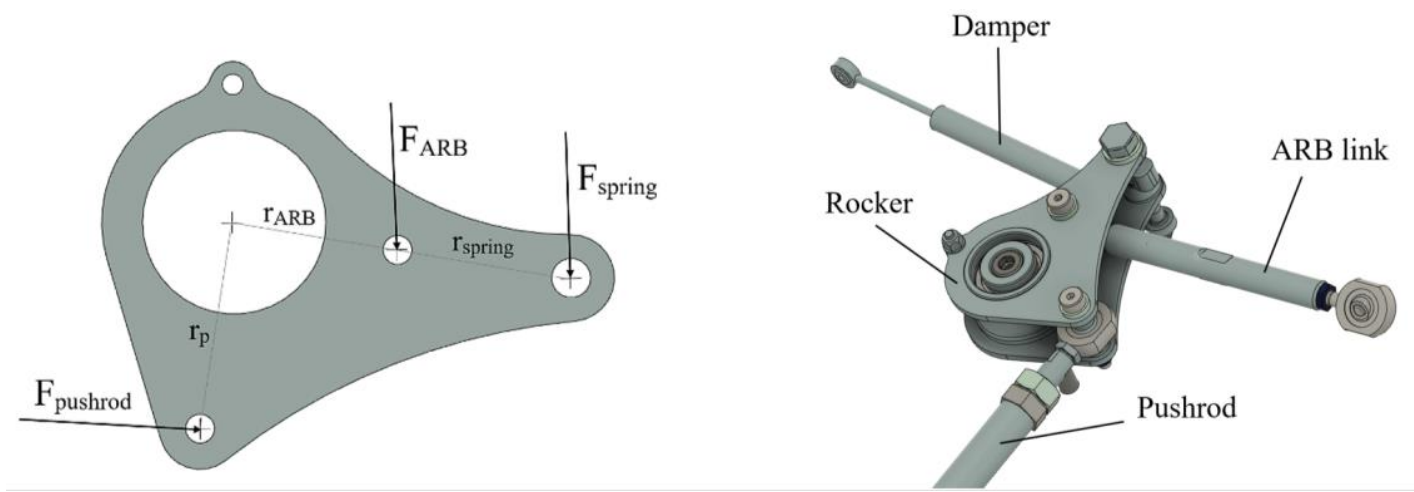

Figure 1. Forces acting on the rocker (left) and the suspension assembly model (right)

Both topology optimization and generative design studies were conducted within the Autodesk Fusion 360 software, the first using a Shape optimization module, and the latter using Generative Design module. The initial model was imported as a STEP file, where in case of Shape optimization only a single part of the rocker plate was needed, while Generative design required the whole assembly of the rocker and all of the connecting parts. The mass of the initial rocker plate was $0.025 \mathrm{~kg}$, and the used material was aluminium 7075-T6. To achieve comparable results, the same criteria i.e. constraints and load case were set up equally in both studies. Value of the push force from a pushrod was $2500 \mathrm{~N}$, which has already been magnified by a safety factor. Hence, generated designs with a safety factor greater than 1 were considered acceptable. Mesh for Shape optimization was generated within the study and was adjusted to fit the design model.

\section{Study workflow}

While preparing the design studies few significant differences were noticed in the study workflows which have an impact on final designs. The main differences are in the initial geometry preparation and design space definition. Additionally, generative design includes a possibility of selecting multiple 
materials and manufacturing methods to perform the study, and also provides possibilities for the comparative overview of the results.

\subsection{Shape optimization study}

Shape optimization module within Autodesk Fusion 360 Simulation was used for conducting topology optimization studies. A topology optimization workflow that was followed is presented in the Figure 2.

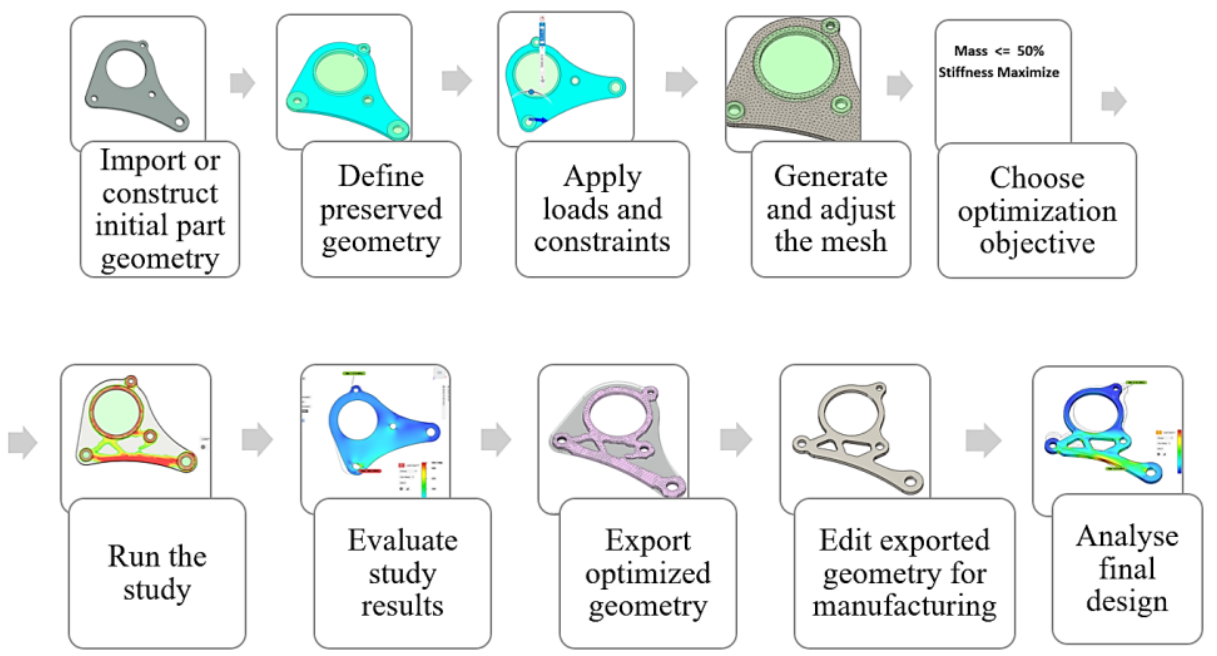

Figure 2. Topology optimization workflow

First, a STEP model of the initial rocker plate was imported into the simulation environment. It represents the outer limits of the design space, meaning that the optimized design will be within that initial volume. A material for the study has to be assigned. Second, it is necessary to define preserved geometry which are typically the features that are in the place of connections with other components. A simplified load model is applied to the component by defining loads and structural constraints. Then the mesh can be generated, which can be further locally adjusted to better fit the desired outcomes. The design can be optimized according to one of the two objectives: mass minimization or stiffness maximization in order to minimize the material usage while maintaining the required strength. The design objective was the reduction of mass up to $50 \%$ of the initial mass, and the chosen study material was aluminium 7075-T6. The result of the topology optimization study is a single most optimized design according to the given objective. Often, the result is an organic shape, therefore the topology optimization workflow usually doesn't end with the export of the generated design, but requires additional editing of the geometry to be properly prepared for manufacturing.

\subsection{Generative design study}

Generative design study requires a different approach from the start. Associated workflow for the generative study is shown in the Figure 3. Unlike topology optimization, generative design method doesn't use the maximum volume as a limitation of the design space. Instead, rather than removing unnecessary material, this method builds up geometry connecting the preserved design areas while avoiding prohibited areas, so called obstacles. Initial geometry can be modelled from scratch in any CAD modeller or can be imported as an existing assembly consisting of parts that that are connected to or present spatial limitations to the design of a target part. If the geometry is designed from scratch, designers need to take a different approach to design space modelling than conventional part design. Design features required for a generative study consist of body fractures that must be kept in the design, but that do not limit a part's final structure, and of design areas that cannot be filled with material. The latter are geometry obstacles which represent other components, space that needs to be left to enable component movement or space to be left for component installation (e.g. bolts and nuts). Initial geometry model can rather quickly be constructed of design primitives such as cubes, cylinders and spheres, or can be imported from already existing model. In our case existing model of the suspension assembly was used. After setting up the starting geometry, loads 
and constraints are applied to the model. As mentioned previously, generative design can simultaneously consider multiple different materials and manufacturing methods, which reduces the number of studies that would need to be conducted by the designer in order to test all the desired materials. Additionally, design objective has to be defined, which is either reduction of mass or maximisation of stiffness. Generative design study results in various designs which satisfy the input data and design objectives in different ways, as different combinations of structure and materials are possible. The algorithm behind the study already considers manufacturing limitations, therefore a number of generated results don't require further redesign.
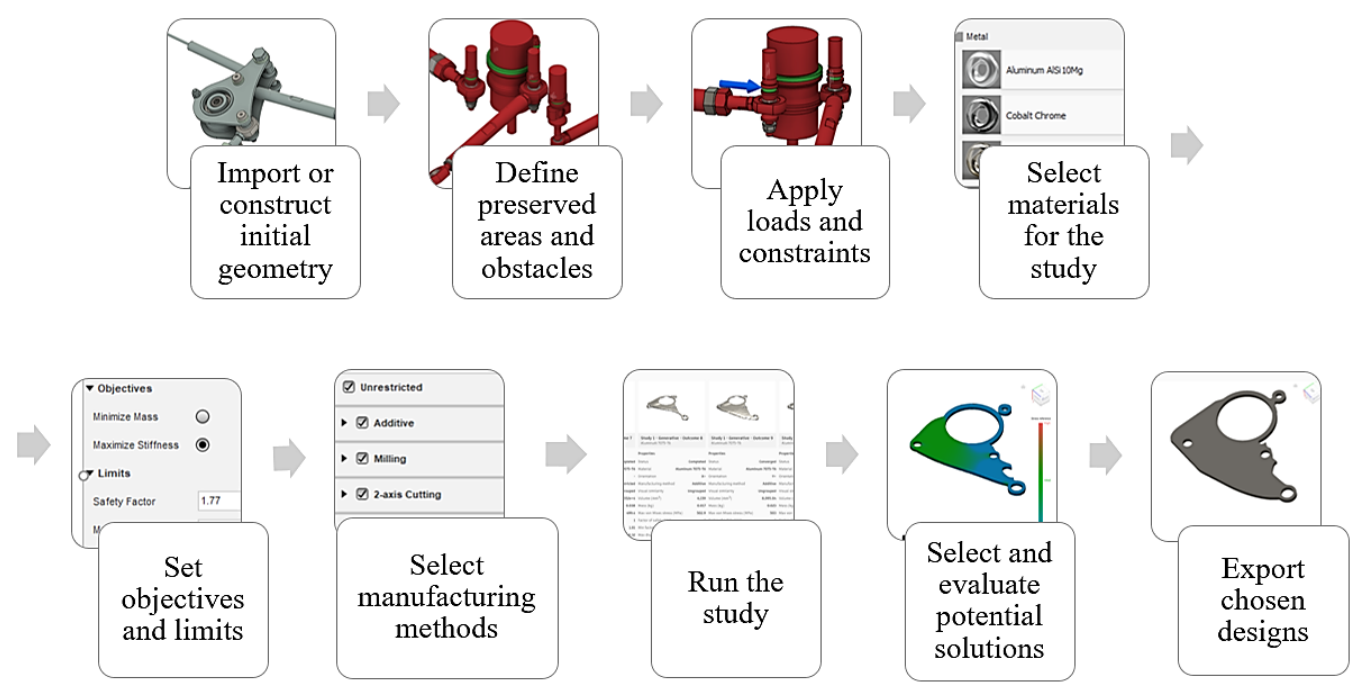

Figure 3. Generative design workflow

The chosen objective of the study was minimizing mass so that the minimum value of the safety factor was 1. For the generative design study four materials were chosen for consideration: Al 7075-T6, AlSi10Mg, Titanium 6Al-4V and Stainless steel AISI 304. In addition to chosen materials, three manufacturing methods were selected to be considered: 2-axis cutting, 3-axis milling, additive manufacturing; also the unrestricted method was included.

\section{Results}

\subsection{Shape optimization}

Shape optimization resulted in a single best solution according to the set criteria. The shape optimization study took less than 2 minutes, but additional time was spent on redesigning the final design geometry. Figure 4 a) shows the optimization results before export to mesh body (Figure 4 b). Red areas represent critical load path, which indicates where the material should be distributed. However, calculation of the critical load path doesn't consider stresses, therefore a stress analysis needs to be conducted on the final design to validate the design's applicability.

a)

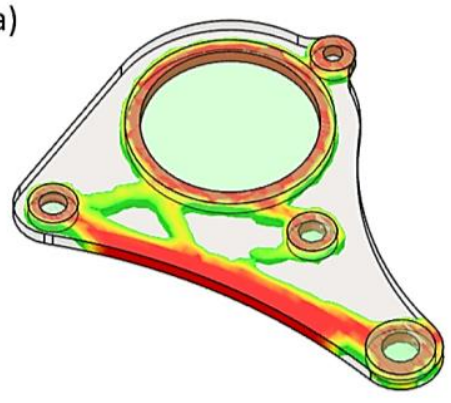

a)

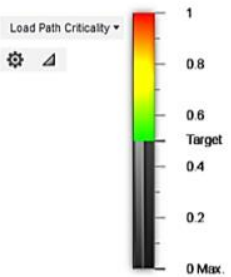

b)

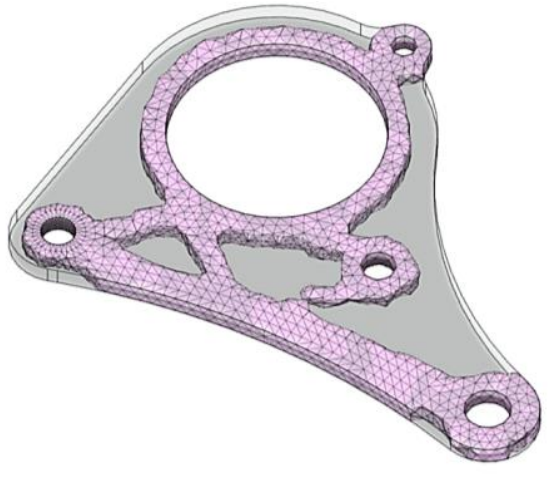

Figure 4. Optimisation result as a) critical load path and b) mesh body 
Final redesigned part is shown on Figure 5. Mass was reduced to 55.6\% of the initial mass, i.e. $45 \%$ reduction in mass. A stress analysis was conducted on the redesigned part, which has implied the necessary changes in the size of radiuses to avoid stress concentrations. Design was edited until the value of von Mises stress was under the yield strength, i.e. safety factor was greater than 1 (Figure 6).
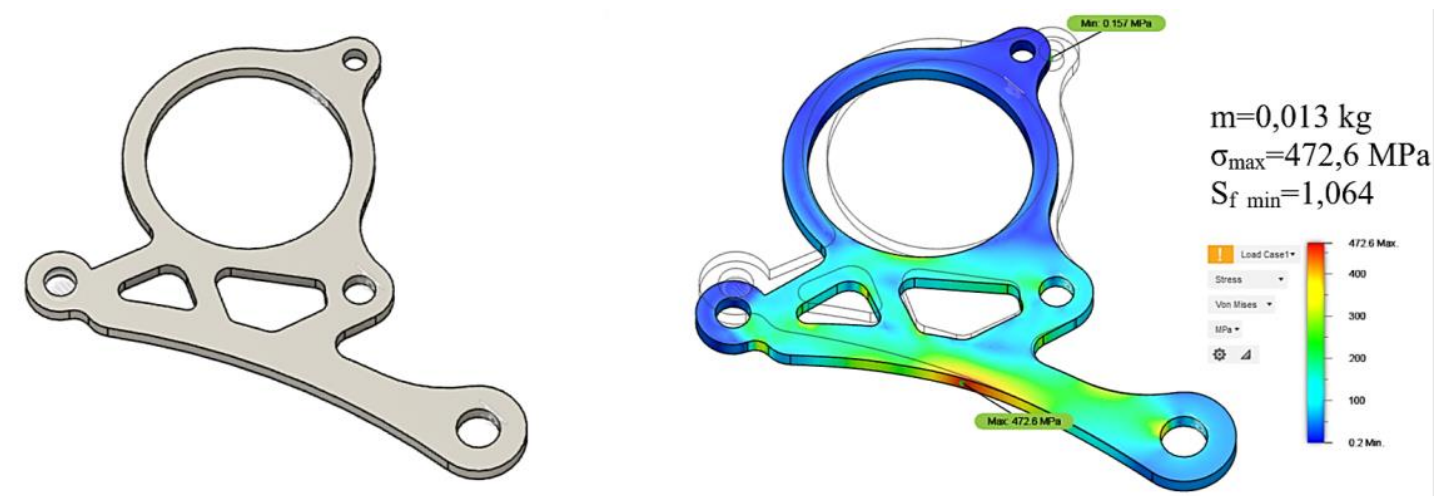

Figure 5. Final redesigned part geometry (left) and stress analysis (right)

\subsection{Generative design}

The most distinguishable results are presented on the Figure 6. The study took 2.5 hours to complete. Only a few generated results were provided that had a good combination of geometry and a manufacturing method, that could be directly exported and produced without additional editing.

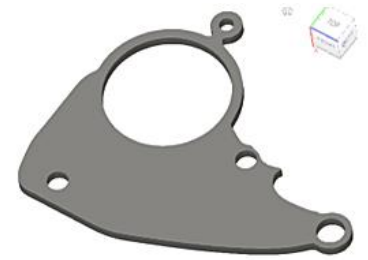

$\mathrm{m}=0,024 \mathrm{~kg}$

$\mathrm{S}_{\mathrm{f}}=1$

Aluminum 7075-T6

2 axis cutting

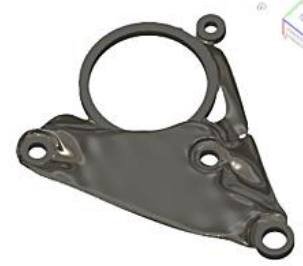

$\mathrm{m}=0,053 \mathrm{~kg}$

$\mathrm{S}_{\mathrm{f}}=1$

Titanium 6Al-4V

Unrestricted

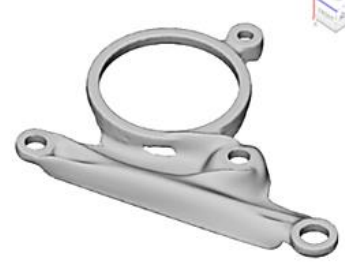

$\mathrm{m}=0,041 \mathrm{~kg}$

$\mathrm{S}_{\mathrm{f}}=1$

Titanium 6Al-4V

3 axis milling

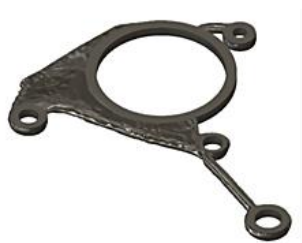

$\mathrm{m}=0,018 \mathrm{~kg}$

$\mathrm{S}_{\mathrm{f}}=1$

Titanium 6Al-4V

Additive

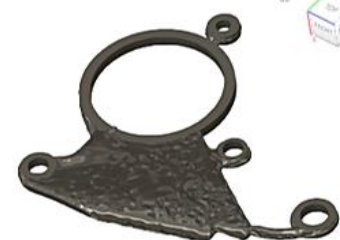

$\mathrm{m}=0,028 \mathrm{~kg}$

$\mathrm{S}_{\mathrm{f}}=1$

Titanium 6Al-4V

Additive

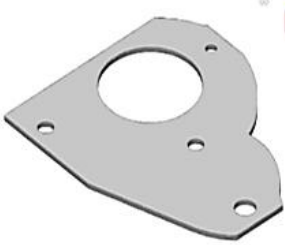

$\mathrm{m}=0,039 \mathrm{~kg}$

$\mathrm{S}_{\mathrm{f}}=1$

Aluminum AlSi10Mg

2 axis cutting

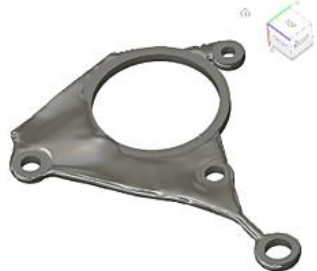

$\mathrm{m}=0,017 \mathrm{~kg}$

$\mathrm{S}_{\mathrm{f}}=1$

Aluminum 7075-T6

Additive

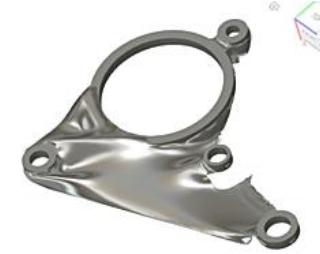

$\mathrm{m}=0,021 \mathrm{~kg}$

$\mathrm{S}_{\mathrm{f}}=1$

Aluminum 7075-T6

3 axis milling

\section{Figure 6. Results of a generative design study}

It is evident that the algorithm used a larger space to generate designs than in the topology optimization study. On the other hand, only on a small number of generated design alternatives the additional holes were added. Some designs had areas with the very small thickness of material, which didn't contribute to the stability of the structure, therefore could have been removed in order to achieve a more optimal form (Figure 7). This resulted in generally higher mass of the generated designs, which indicates there is still space left for improvement of this method. 

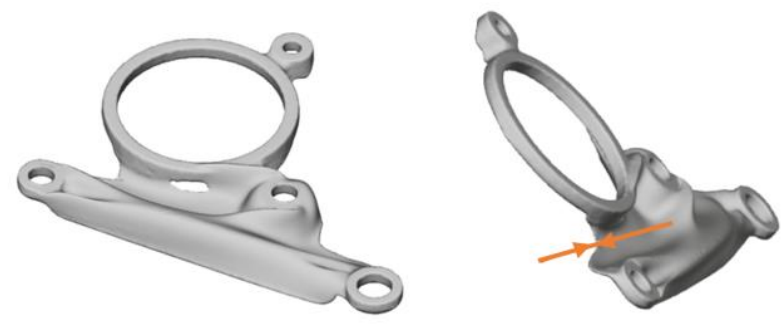

Figure 7. Design areas with small thickness

Autodesk Fusion 360 has the ability of an overview of the design alternatives and their performance according to selected criteria (Figure 8). For studied example it is evident on the graph that the optimal designs with the lowest mass and stress values are made of aluminum. For each design it is possible to view the stress distribution (Figure 8).

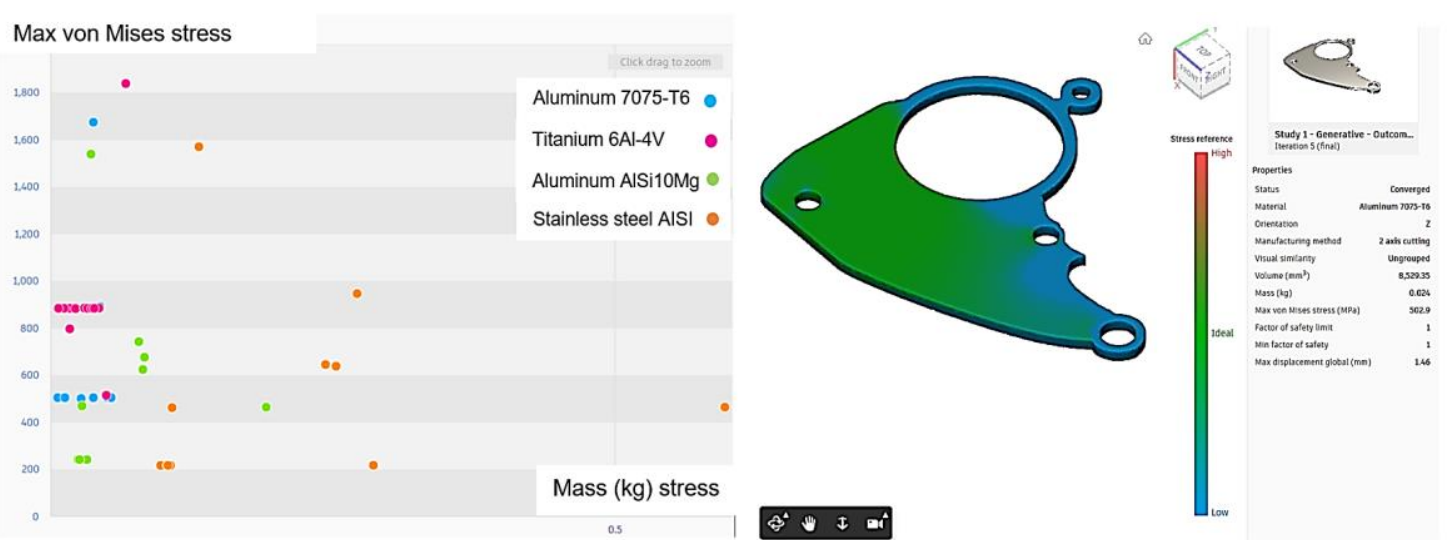

Figure 8. Generative design solution evaluation: design alternatives mapped in the design (left) space, stress analysis of selected design (right)

To show a comprehensive comparison, the following table includes a summarized overview of the study parameters that were set for each study and their results (Table 1).

Table 1. Comparison of study parameters and results

\begin{tabular}{|l|l|l|}
\hline & Topology optimization \\
\hline Initial geometry & & \\
\hline Materials & Solid part of a rocker & \\
\hline Manufacturing methods & Not considered & $\begin{array}{l}\text { 2-axis cutting, 3-axis milling, additive } \\
\text { manufacturing, unrestricted }\end{array}$ \\
\hline Objectives & Mass reduction up to 50\% & Minimize mass, $\mathrm{S}_{\mathrm{f}} \geq 1$ \\
\hline Study result & 1 optimal design, mesh body & \\
\hline & & \\
\hline Mass reduction & $45 \%$ & Max. 32\% \\
\hline Duration of a study & 2 minutes & Available, mass and stress comparison \\
\hline Parallel design comparison & Not available within the tool & Not required in some cases \\
\hline Additional design editing & 30 minutes, required & \\
\hline
\end{tabular}




\section{Discussion}

In the conceptual design phase of product development process designers explore the design space and test different design alternatives to find the most optimal solution. In the recent years powerful computer tools have been developed that support the design process by generating optimal designs which satisfy the set design criteria. In this paper a comparative study of topology optimization and generative design tools was presented. Table 2 gives a short overview of requirements and study parameters for both tools.

Table 2. Requirements and parameters for topology optimization and generative design tools

\begin{tabular}{|c|c|c|}
\hline & Topology optimization & Generative design \\
\hline Initial geometry & $\begin{array}{l}\text { Solid body of a maximum part } \\
\text { volume }\end{array}$ & $\begin{array}{l}\text { Surrounding bodies and/or surfaces } \\
\text { (existing assembly or constructed } \\
\text { parts) }\end{array}$ \\
\hline Design space definition & $\begin{array}{l}\text { Maximum volume and preserved } \\
\text { geometry }\end{array}$ & Obstacles and preserved geometry \\
\hline Number of materials per study & 1 & 1 or more \\
\hline Design for manufacturing & Not included & Several manufacturing methods \\
\hline Study result & Single most optimal design & Multiple optimal designs \\
\hline $\begin{array}{l}\text { Duration of a study } \\
\text { (for equal design tasks) }\end{array}$ & Several minutes & Several hours \\
\hline Parallel design comparison & Not applicable & Mass, stress, visual comparison \\
\hline Additional design editing & Required & Not required for some cases \\
\hline
\end{tabular}

Even though both tools are based on algorithms for topology synthesis, they require rather different approaches. Topology optimization usually applies mesh-dependant optimization methods, such as SIMP, which is broadly investigated and adjusted to the level it provides useful optimal results, however the initial geometry required for the study limits the final shape of generated designs. To tackle this issue from a user perspective, it is possible to set up the initial model as a very large design space, but this process can require significant efforts if there are multiple components with complex geometries surrounding the target design part. Another disadvantage of this tool is that in only produces one design per study. Further research of this method could consider possibilities of parallelisation of multiple studies with variable design parameters, such as e.g. starting geometry or mass reduction rates.

On the other hand, generative design tools based on level set method for optimization do not require fully defined design space, which leaves more possibilities to the algorithm for design variation. However, setting up study geometry for generative design would require engineers to adopt a different way of thinking about and approaching design. Generative design tools are fairly new and their algorithms still require further adjustments to be able to achieve the results of the high quality. There are several issues present with the current method that we discovered: designs containing nonfunctional areas with a rather small material thickness; rare inclusion of additional holes in the structure resulting in excessive material distribution; only a few manufacturing methods available for testing. Another concern related to generative design tools is that they are highly computationally demanding, and studies usually take up to several hours to generate the results. However, having these tools available on could mitigates the necessity for specialized equipment.

Currently neither of the tools considers the cost of materials and manufacturing process, which is one of the major concerns of design engineers, therefore its implementation should be considered for future versions of the tools. More research is required to explore how the implementation of these tools in the early phases would influence current designer's work process.

We argue that generative design tools have greater potential to better respond to the needs of conceptual design phase, primarily because they are able to simultaneously test a larger number of materials and generate multiple design alternatives, which can save time and support greater creativity. However, generative design tools are still in development and consideration of a few larger issues that we 
mentioned is needed before they become broadly used by engineers. In the conceptual design phase, topology optimization is a better choice for optimization of already existing components, whereas generative design provides more support when design layout and dimensions are not yet defined. Nevertheless, it is possible to use topology optimization tools to get the initial idea on how to navigate the design, but current tools will only provide the designers with a single optimal solution for one study. It is important to outline that both topology optimization and generative design results will greatly depend on the design space definition, setup of the loads and constraints, as well as on the set objectives. Hence, to make use of these methods the designers need to be skilled in defining a model for optimization to be able to set up the design studies and evaluate the generated results.

\section{References}

Anderson, D.M. (2014), Design for Manufacturability: How to Use Concurrent Engineering to Rapidly Develop Low-Cost, High-Quality Products for Lean Production, CRC Press.

Bendsøe, M.P. (1989), "Optimal shape design as a material distribution problem", Structural Optimization, Springer-Verlag, Vol. 1 No. 4, pp. 193-202. https://doi.org/10.1007/BF01650949

Bendsøe, M.P. and Kikuchi, N. (1988), "Generating optimal topologies in structural design using a homogenization method", Computer Methods in Applied Mechanics and Engineering, North-Holland, Vol. 71 No. 2, pp. 197-224. https://doi.org/10.1016/0045-7825(88)90086-2

Bici, M., Broggiato, G.B. and Campana, F. (2017), "Topological Optimization in Concept Design: starting approach and a validation case study", Springer, pp. 289-299. https://doi.org/10.1007/978-3-319-45781-930

Caldas, L.G. (2001), An evolution-based generative design system: using adaptation to shape architectural form, [PhD Thesis], Massachusetts Institute of Technology.

Chen, X. "Anthony" et al. (2018), "Forte: User-Driven Generative Design", Proceedings of the 2018 CHI Conference on Human Factors in Computing Systems 2018, ACM Press, New York, New York, USA, pp. 1-12. https://doi.org/10.1145/3173574.3174070

Deb, K. (2011), Multi-Objective Optimization Using Evolutionary Algorithms: An Introduction, available at: http://www.iitk.ac.in/kangal/deb.htm (accessed 4 July 2019).

Horváth, I. (2004), “On some Crucial Issues of Computer Support of Conceptual Design”, Product Engineering, Kluwer Academic Publishers, Dordrecht, pp. 123-142. https://doi.org/10.1007/1-4020-2933-0_9

Jia, H. et al. (2011), "Evolutionary level set method for structural topology optimization", Computers and Structures, Vol. 89 No. 5-6, pp. 445-454. https://doi.org/10.1016/j.compstruc.2010.11.003

Kazi, R.H. et al. (2017), "DreamSketch: Early Stage 3D Design Explorations with Sketching and Generative Design", Proceedings of the 30th Annual ACM Symposium on User Interface Software and Technology UIST '17, ACM Press, New York, New York, USA, pp. 401-414. https://doi.org/10.1145/3126594.3126662

Krish, S. (2011), “A practical generative design method”, Computer-Aided Design, Vol. 43 No. 1, pp. 88-100. https://doi.org/10.1016/j.cad.2010.09.009

Meyer, A.W. and Vajna, S. (2018), "Support of searching for solutions by automated structural optimization", pp. 369-380. https://doi.org/110.21278/idc.2018.0383

Querin, O.M. (2017), Topology Design Methods for Structural Optimization, Academic Press.

Rozvany, G. (2000), "The SIMP method in topology optimization - Theoretical background, advantages and new applications", https://doi.org/10.2514/6.2000-4738

Shea, K., Aish, R. and Gourtovaia, M. (2005), "Towards integrated performance-driven generative design tools", Automation in Construction, Vol. 14 No. 2, pp. 253-264. https://doi.org/10.1016/j.autcon.2004.07.002

Tejani, G.G. et al. (2018), "Size, shape, and topology optimization of planar and space trusses using mutationbased improved metaheuristics", Journal of Computational Design and Engineering, Elsevier, Vol. 5 No. 2 , pp. 198-214. https://doi.org/10.1016/J.JCDE.2017.10.001

Tyflopoulos, E. et al. (2018), "State of the art of generative design and topology optimization and potential research needs", DS 91: Proceedings of NordDesign 2018, Linköping, Sweden, 14-17 August 2018.

Vajna, S. et al. (2005), "The Autogenetic Design Theory: An evolutionary view of the design process", Journal of Engineering Design, Vol. 16 No. 4, pp. 423-440. https://doi.org/10.1080/09544820500267781

Wang, L. et al. (2002), Collaborative Conceptual Design - State of the Art and Future Trends, available at: www.elsevier.com/locate/cad (accessed 15 November 2019).

Xie, Y.M. and Steven, G.P. (1994), "Optimal design of multiple load case structures using an evolutionary procedure”, Engineering Computations, Vol. 11 No. 4, pp. 295-302. https://doi.org/10.1108/0264440 9410799290 